Supporting information

\title{
Enhancing Persistent Luminescence of Cellulose by Dehydration for Label-free Time-resolved Imaging
}

Zece Zhu*, Lifan Zeng, Wei Li, Di Tian*, Weilin Xu

Department of Chemistry and Chemical Engineering, Hubei Key Lab of Biomass Fibers and Eco-dyeing \& Finishing, State Key Laboratory of New Textile Materials and Advanced Processing Technologies, Wuhan Textile University, Wuhan 430200, China.

E-mail: dtian@wtu.edu.cn (D. Tian), zczhu@wtu.edu.cn (Z.Zhu)

Number of pages: 6

Number of figures: 12

Number of tables: 1

\section{Content}

Determination of luminescence quantum yield............................................... 2

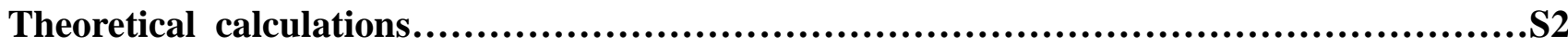

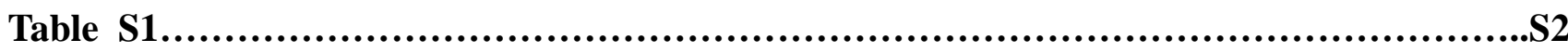

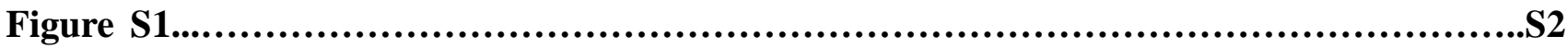

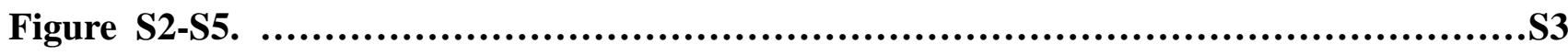

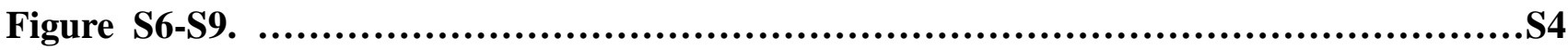

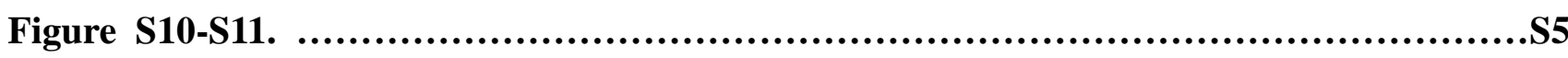

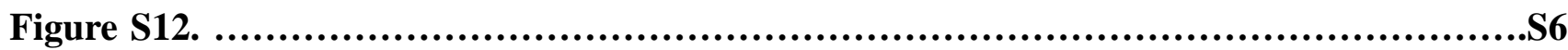

References.......................................................................................... 
Determination of luminescence quantum yield: The absolute luminescence quantum yields were measured according to the references [1,2]. A custom designed integrating sphere (Jinan gen spectrum instrument co., LTD) with an inner diameter of $50 \mathrm{~mm}$ was used to measure the luminescence quantum yield of cellulose samples. The cellulose powder was filled into a quartz tube, which was interted in the integrating sphere, excited by a $310 \mathrm{~nm}$ LED (Shenzhen Chundaxin Photoelectric Co., Ltd) with a narrow band filter (BP317-20nm, Rayan Technology Co., Ltd). A spectrometer (NOVA, Ideaoptics, Inc.) were used for measuring the luminescence spectra. The wavelength-dependent spectral responsivity of the system was calibrated by a tungsten halogen lamp. The wavelength dependence of radiance of the lamp was calibrated by a spectral irradiance colorimeter (OHSP350UV, Hangzhou Hopoo Light\&Color Technology Co.,Ltd.).

Theoretical calculations: The original geometry of two cellotriose chains were extracted from a crystal structure of Cellulose I $\alpha$ reported in the reference [3-5]. A serial of DFT and TDDFT calculations of cellotriose chains and the cellotriose chains with the existence of water were optimized by TDDFT method at B3LYP/6-31+G(d) using Gaussion 09 software.

Table S1. The absolute luminescence quantum yields of cellulose.

\begin{tabular}{lll}
\hline & Normal $^{\mathrm{a}}$ & Dried \\
\hline$\alpha$-cellulose & $3.3 \%$ & $4.3 \%$ \\
MCC & $2.9 \%$ & $4 \%$ \\
$\mathrm{CNC}$ & $0.9 \%$ & $1.5 \%$ \\
\hline
\end{tabular}

${ }^{a}$ Water absorption would greatly affect the luminescence quantum yields, which may cause much deviation.
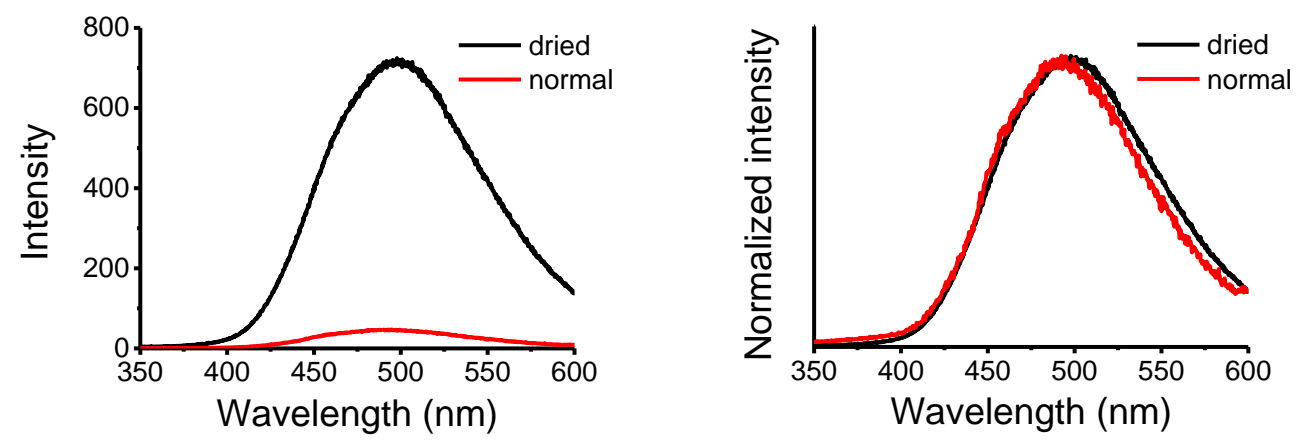

Figure S1. Phosphorescence spectra of dried and normal $\alpha$-cellulose at room temperature. $\lambda_{\mathrm{ex}}=310 \mathrm{~nm}$. 

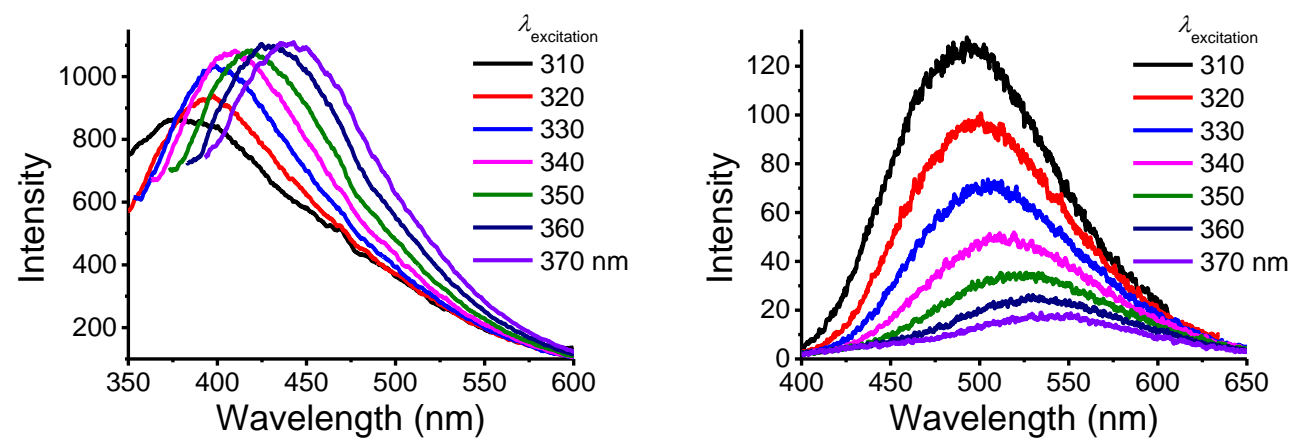

Figure S2. Fluorescence and phosphorescence spectra of dried MCC excited at different wavelength.
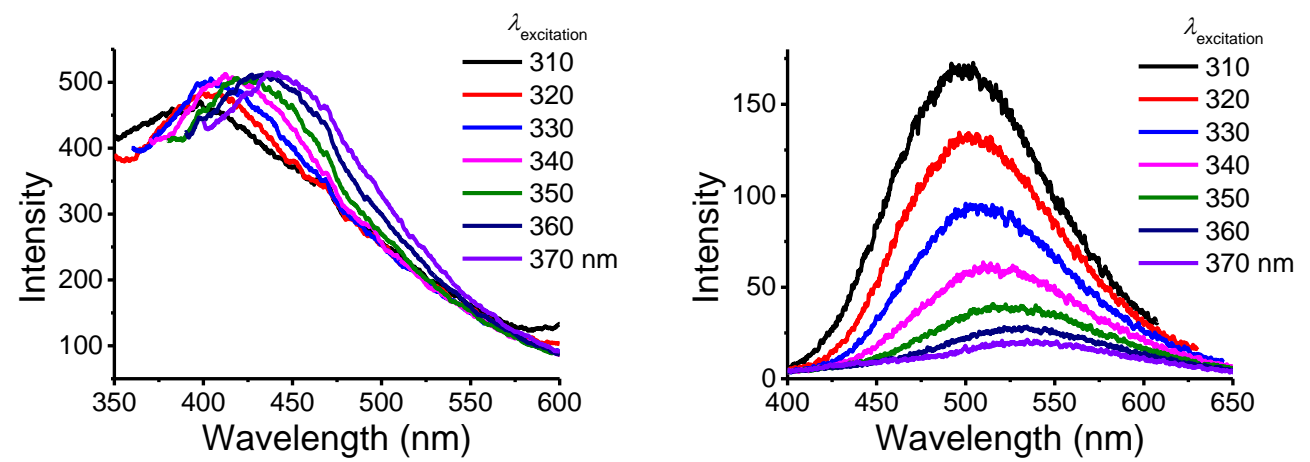

Figure S3. Fluorescence and phosphorescence spectra of dried CNC excited at different wavelength.
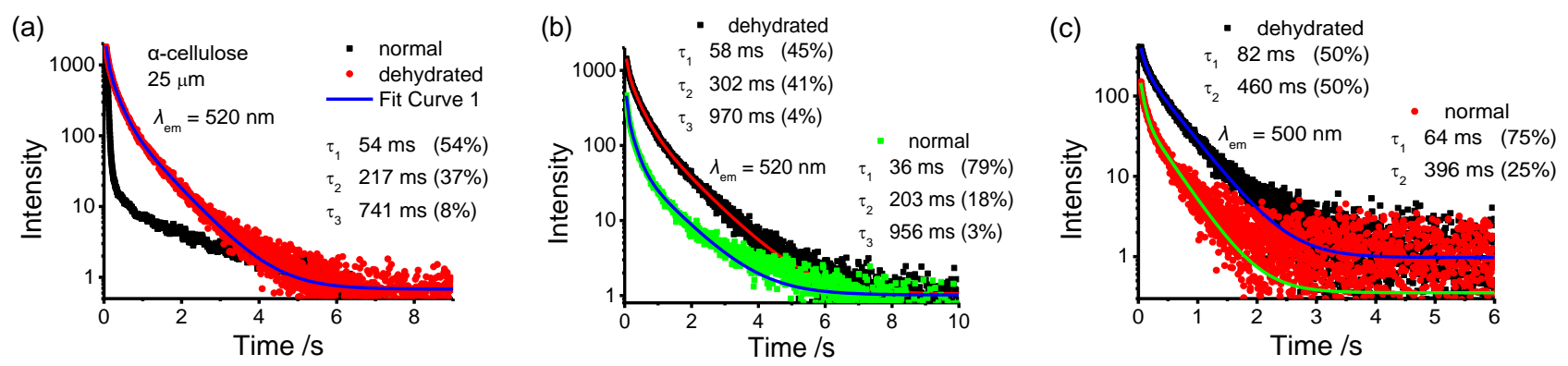

Figure S4. Luminescence decays of $\alpha$-cellulose (a), MCC (b) and CNC (c) before (black) and after (red) thermal dehydration at room temperature. $\lambda_{\mathrm{ex}}=310 \mathrm{~nm}$.

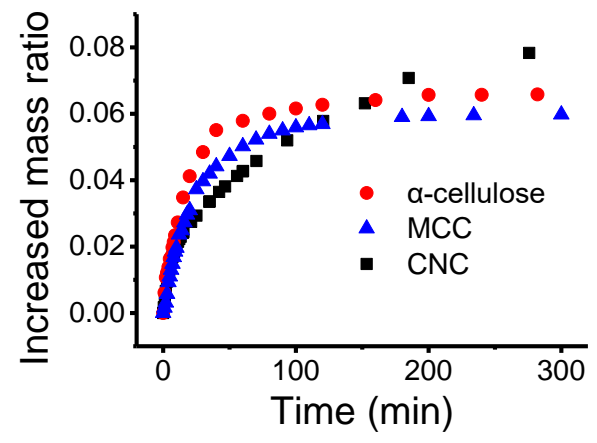

Figure S5. The mass variation of $\alpha$-cellulose, MCC and CNC exposed to air at room temperature after thermal dehydration. 

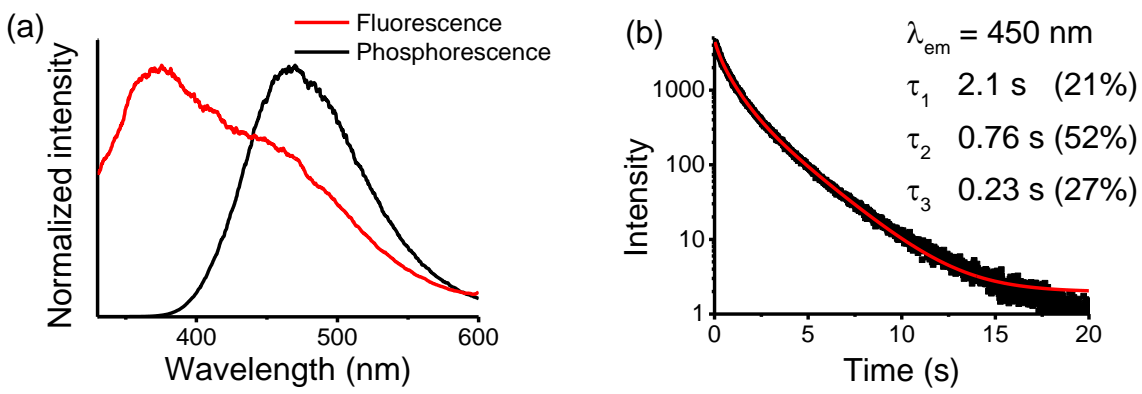

Figure S6. Luminescence spectra (a) and decay (b) of $\alpha$-cellulose at $77 \mathrm{~K}$. $\lambda_{\mathrm{ex}}=310 \mathrm{~nm}$.

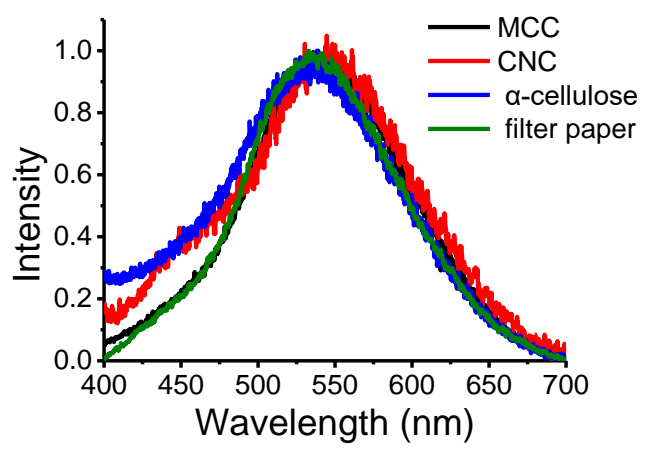

Figure S7. Phosphorescence spectra of dried cellulose materials. $\lambda_{\mathrm{ex}}=370 \mathrm{~nm}$.
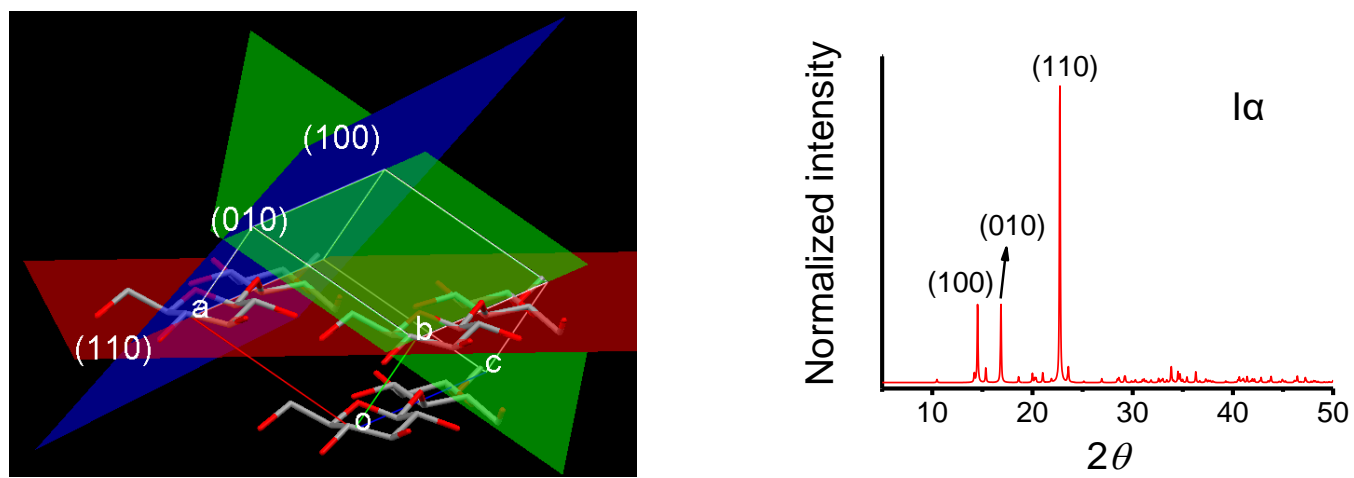

Figure S8. Crystal structure of cellulose I $\alpha$ and its simulated X-Ray powder diffraction pattern, according to a revised .cif file in the reference [5].
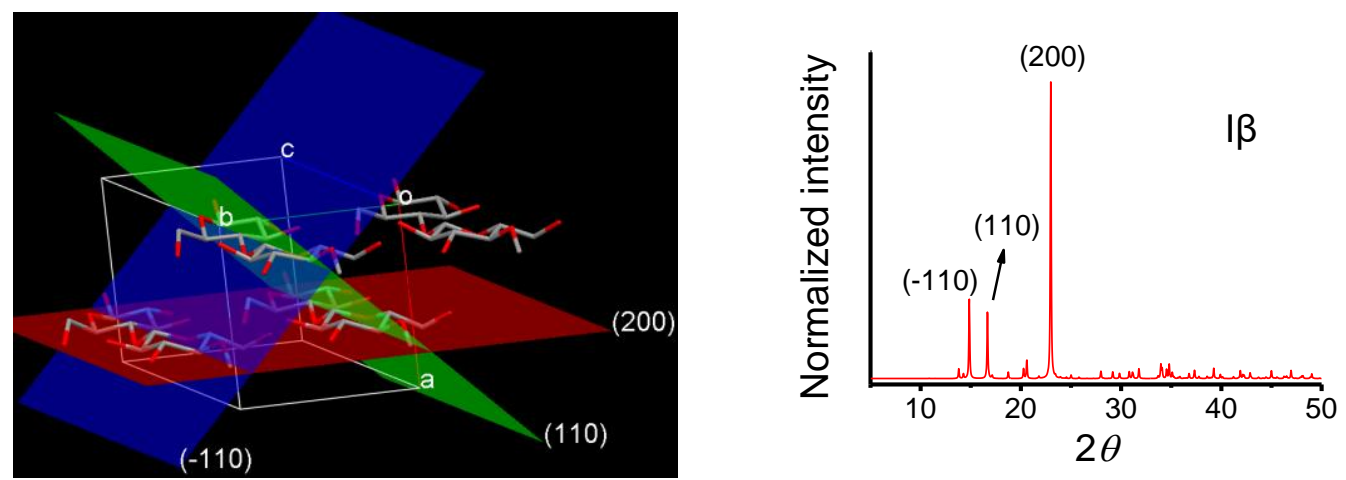

Figure S9. Crystal structure of cellulose I $\beta$ and its simulated X-Ray powder diffraction pattern, according to a revised .cif file in the reference [5]. 


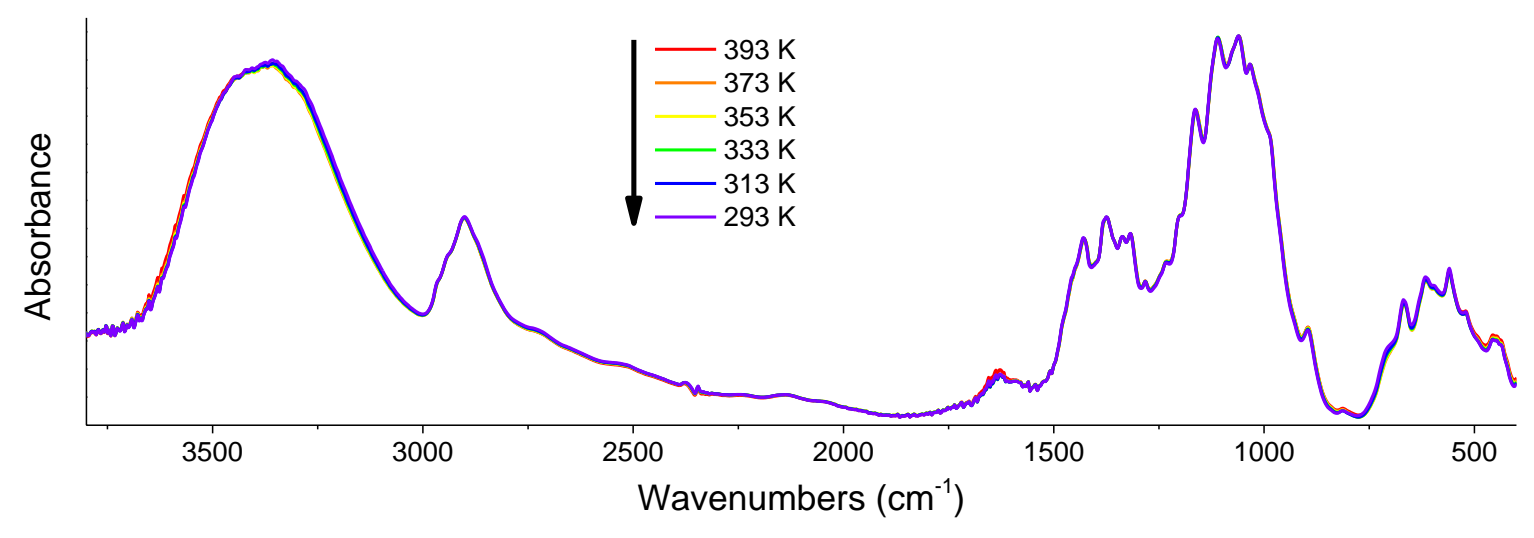

Figure S10. Reflectance infrared spectra of $\alpha$-cellulose at temperature from 393 to $293 \mathrm{~K}$.

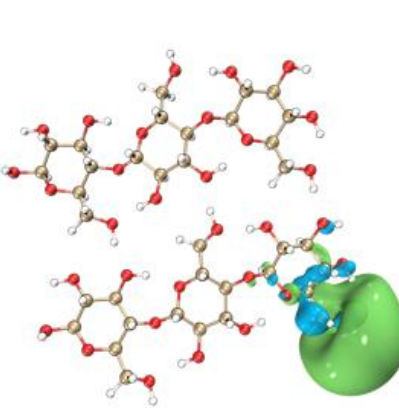

LUMO

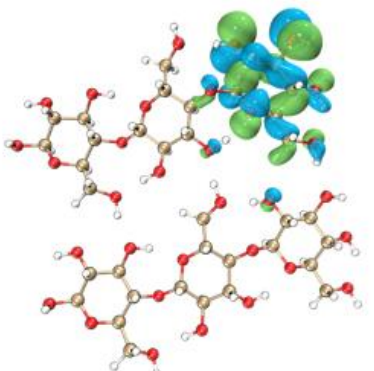

HOMO

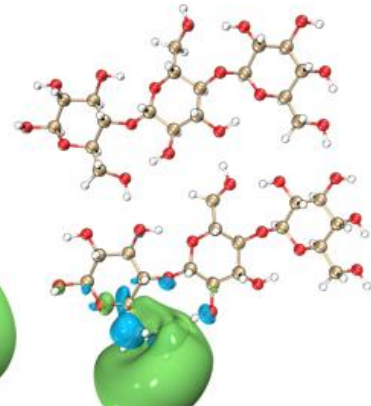

LUMO+1

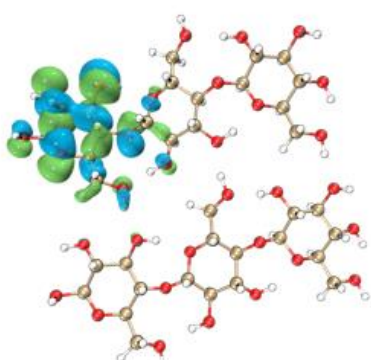

HOMO-1

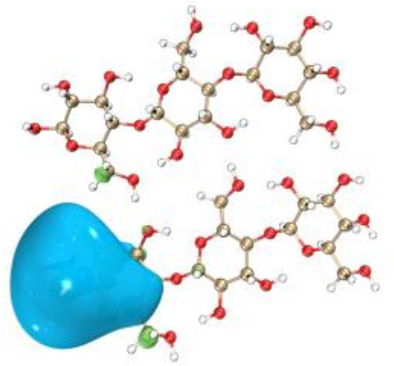

LUMO+2

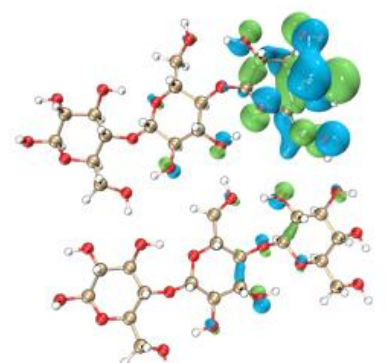

HOMO-2

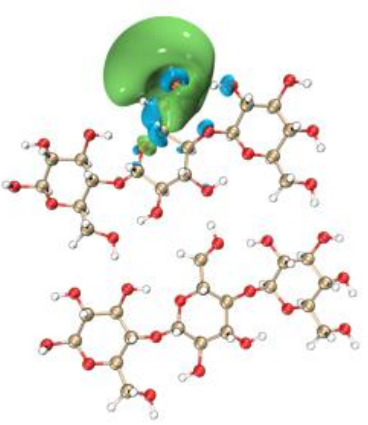

LUMO+3

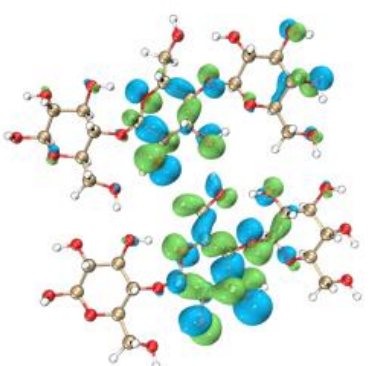

HOMO-3

Figure S11. Molecular orbitals of the optimized ground state configuration of cellulose. 


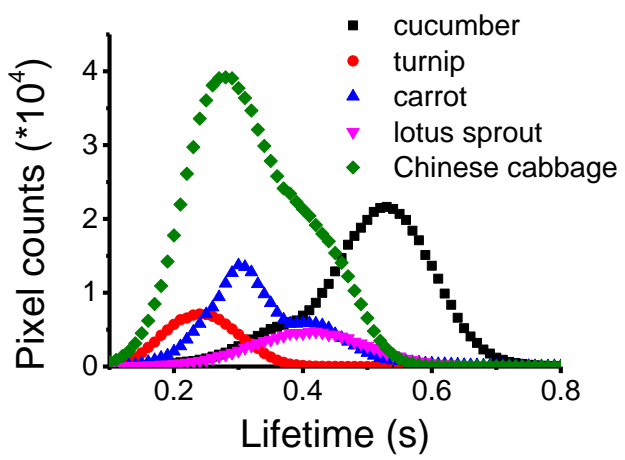

Figure S12. Statistical distribution of luminescence lifetime values of plant tissue imaging in Figure 10.

\section{References}

[1] M. Kaiser, C. Würth, M. Kraft, I. Hyppänen, T. Soukka, U. Resch-Genger, Nanoscale, 2017, 9, 10051-10058.

[2] N. Yanai, K. Suzuki, T. Ogawa, Y. Sasaki, N. Harada, N. Kimizuka, J. Phys. Chem. A 2019, 123, 10197-10203.

[3] Y. Nishiyama, P. Langan, H. Chanzy, J. Am. Chem. Soc. 2002, 124, 9074-9082;

[4] Y. Nishiyama, J. Sugiyama, H. Chanzy, P. Langan, J. Am. Chem. Soc. 2003, 125, 14300-14306.

[5] A. D. French, Cellulose 2014, 21, 885-896. 